\title{
MEJORA DEL PROCESO DE FABRICACIÓN DE ESTIBAS DE MADERA: UN CASO DE ESTUDIO
}

Juan Carlos Herrera-Vega ${ }^{1}$, Germán Herrera-Vidal ${ }^{2}$, Cindy Isabel González-Polo ${ }^{3}$

\author{
${ }^{1}$ Magíster en Ingeniería Industrial. Profesor, Ingeniería Industrial. Correo electrónico: \\ jherrerav@tecnocomfenalco.edu.co \\ ${ }^{2}$ Magister en Ingeniería Industrial. Profesor, Ingeniería Industrial \\ ${ }^{3}$ Ingeniero industrial \\ Fundación Universitaria Tecnológico Comfenalco, Cartagena de Indias, Colombia
}

Fecha de recibido: 20 de abril del 2017

Fecha de aprobado: 18 de agosto del 2017

Cómo citar este artículo: J. C. Herrera-Vega, G. Herrera-Vidal y C. González-Polo, "Mejora del proceso de fabricación de estibas de madera. Un caso de estudio", Ingeniería Solidaria, vol. 13, n. ${ }^{\circ} 23$, pp. 40-55, Sept. 2017. doi: https://doi. org/10.16925/in.v23i13.2004

Resumen. Introducción: el artículo muestra los resultados del proyecto de investigación aplicada sobre "Diseño de mejora al proceso de fabricación de estibas de madera a través de herramientas para el incremento de la productividad. Caso: sector maderero", desarrollado en la Fundación Universitaria Tecnológico Comfenalco, Cartagena, Colombia (2014). Su objetivo fue generar y aplicar acciones de mejora al proceso productivo de la empresa estudiada a partir de herramientas propias de la ingeniería industrial que permitieran un mejor uso de recursos. $\mathrm{Me}$ todología: se aplicaron métodos y metodologías para el incremento de la productividad, como el estudio de métodos y tiempos y la metodología de 5S, una guía para implementar un programa de producción más limpia PML y para el cálculo de rendimiento de la materia prima. Resultados: se estandarizó y mejoró el proceso productivo a partir del estudio de métodos y $5 \mathrm{~S}$, se implantó un estándar razonable de producción para empresa y trabajadores, se evaluó una metodología para calcular rendimientos de la madera y se desarrolló una propuesta para implementar un programa de PML, cuyos lineamientos mejoraron las prácticas operativas. Conclusión: no medir un proceso es tan grave como medirlo erróneamente; esto puede conducir a implantar estándares incumplibles o muy laxos, así como a un clima laboral que genera bajos niveles de productividad debido a los niveles de satisfacción de los empleados por exigencias inadecuadas o al relajamiento de los empleados.

Palabras clave: estibas, estudio del trabajo, manufactura esbelta, metodología 5S, producción más limpia. 


\title{
IMPROVING THE MANUFACTURING PROCESS OF WOODEN PALLETS: A CASE STUDY
}

\begin{abstract}
Introduction: This article shows the results of the applied research project "Improved design of the manufacturing process of wooden pallets through tools for increasing productivity. Case: Wood industry", conducted at the Fundación Universitaria Tecnológico Comfenalco Cartagena during 2014. The objective of the research was to create and apply improvement actions to the productive process of the company studied based on industrial engineering tools that would allow a better use of available resources. Methodology: Methods and methodologies were applied to increase productivity, such as the methods-time measurement and the $5 \mathrm{~S}$ methodology, a guide to implement a cleaner production (CP) program and to calculate the yield of raw materials. Results: The production process was standardized and improved based on the methods-time measurement and 5S. A reasonable production standard was established for the company and its workers. A methodology to calculate wood yields was assessed. A proposal was prepared to implement a CP program, whose guidelines improved the operating practices. Conclusion: Not measuring a process is as serious as measuring it wrongly, since it can lead to the implementation of non-compliant or very lax standards, as well as to a work environment with low levels of productivity due to employee dissatisfaction with inadequate demands, or to the relaxation of employees. Therefore, work study today is highly valid and applicable.
\end{abstract}

Keywords: pallets, work study, lean manufacturing, $5 \mathrm{~S}$ methodology, cleaner production.

\section{MELHORIA DO PROCESSO DE FABRICAÇÃO DE}

\section{PALLETS DE MADEIRA: UM CASO DE ESTUDO}

Resumo. Introdução: o artigo mostra os resultados do projeto de pesquisa aplicada sobre "Design de melhoria do processo de fabricação de pallets de madeira através de ferramentas para o aumento da produtividade. Caso: setor madeireiro", desenvolvido na Fundação Universitária Tecnológico Comfenalco Cartagena durante 2014. O objetivo da pesquisa foi gerar e aplicar ações de melhoria ao processo produtivo da empresa estudada a partir de ferramentas próprias da engenharia industrial que permitiram uma melhor utilização dos recursos disponíveis. Metodologia: foram aplicados métodos e metodologias para o aumento da produtividade, tais como o estudo de métodos e tempos e a metodologia de 5S, um guia para implementar um programa de produção mais limpa PML e para o cálculo de rendimento da matéria-prima. Resultados: o processo produtivo foi padronizado e melhorado a partir do estudo de métodos e 5S. Foi implantado um padrão razoável de produção para empresa e trabalhadores. Foi avaliada uma metodologia para calcular rendimentos da madeira. Foi desenvolvida uma proposta para implementar um programa de PML, a partir de cujos alinhamentos as práticas operativas foram melhoradas. Conclusão: não medir um processo é tão grave quanto medi-lo erroneamente, já que pode levar a implantar padrões difíceis de cumprir ou muito frouxos, assim como um clima laboral que gera baixos níveis de produtividade pelos níveis de satisfação dos empregados por exigências inadequadas, ou ao relaxamento dos empregados, ambos conducentes à diminuição da produtividade razão pela qual o estudo do trabalho tem atualmente uma alta validade e aplicação.

Palavras-chave: pallets, estudo do trabalho, manufatura esbelta, metodologia 5S, produção mais limpa. 


\section{Introducción}

Colombia cuenta con 24805854 ha aptas para desarrollo de proyectos de plantaciones forestales, distribuidas en varios pisos altitudinales. De estas, 7258442 ha tienen una aptitud alta; $6217697 \mathrm{ha}$, una aptitud media, y $11329715 \mathrm{ha}$, una aptitud baja [1]. No obstante la gran oportunidad mencionada anteriormente y la gran disponibilidad de materia prima para poder satisfacer adecuadamente la demanda requerida, las PYME del sector dedicadas a la transformación de productos de madera y corcho, exceptuando muebles, y en particular las dedicadas a la fabricación de estibas de madera, padecen serios problemas que les impiden asegurar la permanencia en el mercado, debido a que no cuentan con estrategias que mejoren sus procesos y su productividad. Lo anterior se hace evidente al evaluar el comportamiento porcentual del indicador de aporte al PIB dentro de la industria manufacturera, de alrededor del $0,1324 \%$, un valor bajo si se compara con el obtenido por el subsector de fabricación de papel, cartón y productos de papel, y cartón que es de 0,4901\% para el 2016 [2]. Sin embargo, al analizar su comportamiento internamente se ve que los rubros de transformación de madera y corcho, y fabricación de madera muestran variaciones positivas de crecimiento desde el 2012, lo cual es muy significativo como mejora de los procesos (figura 1).

Dentro de este contexto, queda claro que el aporte al PIB por parte de cada uno de los sectores de la economía se ve directamente afectado, positiva o negativamente, por el desempeño de las diferentes organizaciones que lo conforman y por la visión de quienes las gerencian. Kaplan y Norton [4], integrando la estrategia y las operaciones para lograr ventajas competitivas, mencionan que es imposible implementar una estrategia visionaria si esta no está relacionada con excelentes procesos operativos y de gobierno. A su vez, la excelencia puede reducir tiempos en procesos y plazos, por lo que es entendible que para poder lograr un mejoramiento del pib es necesario que las empresas de los diferentes sectores de la industria adopten estrategias para la consecución de objetivos que permitan incrementar la competitividad.

Por otra parte, Navas y Marbelís [5] señalan que una empresa debe implantar medidas que le permitan ser más competitiva y eficiente económica y financieramente, de tal forma que haga superior uso de sus recursos para mejorar la productividad y obtener mejores resultados con menores costos de producción; esto es coherente con lo mencionado por Porter [6], quien afirma que la verdadera competitividad se define por la productividad con la que un país utiliza sus recursos humanos, económicos y naturales.

Del mismo modo, Rochina, Mañez y Sanchis-Llopis [7] concluyen que las empresas que implementan procesos de innovación disfrutan de una mayor productividad que las que no lo hacen. Este aumento de la productividad también depende del tamaño de la empresa, ya que las empresas pequeñas ven aumentada su productividad al implementar un nuevo proceso con buenas prácticas, incluso en el mismo año de su instauración, y que, por el contrario, las grandes firmas pueden tardar hasta dos años en

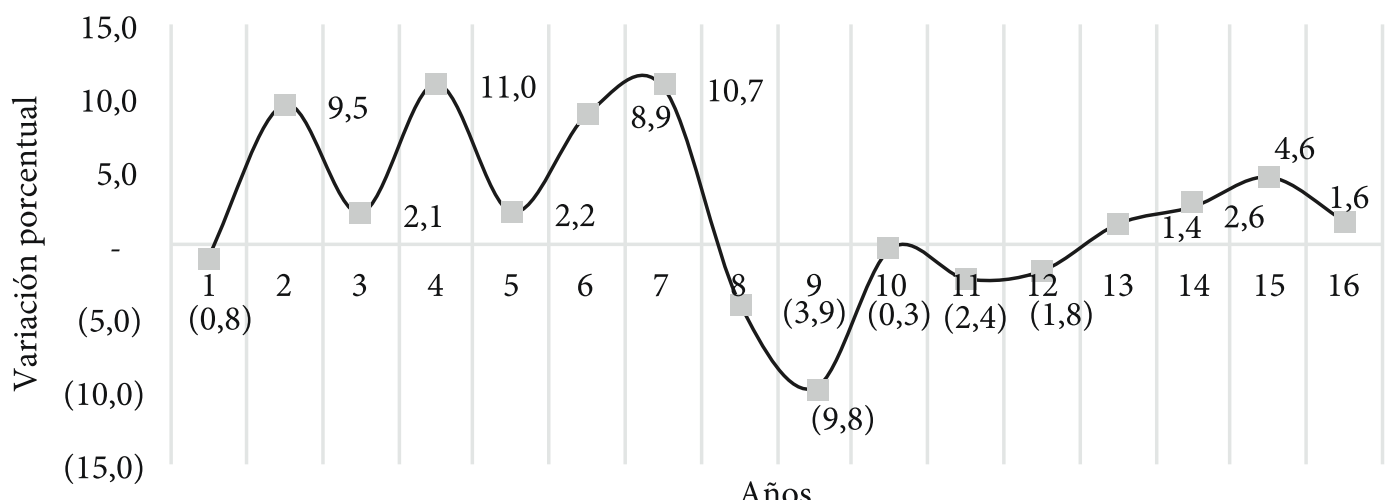

Figura 1. Variación PIB transformación productos de madera y corcho 2000-2016 
ver los efectos positivos de la implantación del nuevo proceso sobre el aumento de su rendimiento.

En este marco de innovación, competitividad, productividad y mejoramiento de los procesos productivos o de manufactura, Urgal-González y García-Vásquez [8] ratifican las ideas de que hay un potencial en la función producción como fuente de ventaja competitiva. De esta forma, la ingeniería industrial como disciplina aporta diversidad de herramientas, metodologías o filosofías de mejora de la productividad que pueden ser aplicadas en diversas empresas de diferentes sectores de la economía. A partir de los diferentes tipos de industrias, procesos y objetivos en la mejora del desempeño, a través de los años se han desarrollado numerosas metodologías dirigidas al sector manufacturero y de servicios. Dentro de ellas se mencionan la manufactura esbelta o lean manufacturing y, como complementarias a esta, las $5 \mathrm{~S}$ y la ingeniería de métodos y tiempos.

Pavnascar y Gerhenson [9] clasifican al menos 101 herramientas esbeltas, tomando como fuente la literatura publicada e inédita, y clasificándola acorde a su utilización, esto es, si son para la identificación, medición o eliminación del desperdicio o muda. Cruelles [11] afirma que con la eliminación o reducción de las actividades innecesarias se disminuye hasta un $50 \%$ en costos de producción, inventarios y tiempos de entrega, además de mejorar la calidad y aumentar la eficiencia del equipo de trabajo, en la medida que se logra identificar el despilfarro o ineficiencias de los procesos. También afirma que de acuerdo a los resultados de sus trabajos que aplicaban las herramientas mencionadas anteriormente se puede obtener una mejora de entre 20 y $50 \%$ de la productividad industrial. Rohani y Zahraee [12] mencionan que el principal objetivo de la lean manufacturing es determinar y eliminar las mudas o desperdicios, así como para lograr ser más competitivas deben mejorar su productividad del sistema y la calidad del producto.

Diversos autores han mostrado la utilidad de las herramientas lean en el mejoramiento de la productividad. Indrawati y Ridwansyah [13] aplicaron estas herramientas en la industria del acero como estrategia para el mejoramiento de la capacidad de producción vía eliminación de mudas o actividades que no agregan valor. Rivas, Romero, Domínguez y Espinosa [14] abordaron la implementación de la metodología de las $5 \mathrm{~S}$ en los laboratorios de la escuela de Ingeniería Industrial. A través del desa- rrollo de la investigación se logró crear una cultura de la organización, así como por medio de la aplicación de las 5S. Bevilacqua, Ciarapica, De Sanctis, Mazzuto y Paciarotti [15] integró las estrategias de las $5 \mathrm{~S}$ en una planta farmacéutica. Implementaron varias técnicas que llevaron a mejoras dramáticas en el cambio tiempo con menos variabilidad. Gupta y Jain [16] describieron cómo identificar equipos que pudieran implementar las prácticas de 5S, así como generar métodos de recolección de datos para asegurarse de la correcta aplicación de estas.

Igual de importantes que las herramientas son las personas y la cultura empresarial. Ramdass [17] encontró que la aplicación de los principios de las $5 S$ puede convertirse en una ventaja competitiva si esta se hace dentro de la cultura organizacional, ya que se deriva en reducción del tiempo perdido, mejora de la productividad y la calidad, entre otras. Dombrowski y Mielke [18] destacaron el liderazgo esbelto como el vínculo perdido entre las herramientas lean, el aprendizaje y el mejoramiento continuo, lo cual es reafirmado por Mann [19], quien afirma que el vínculo perdido es el ajuste entre los comportamientos de liderazgo y las estructuras que lo conforman. Alhuraish, Robledo y Kobi [20] encontraron que las prácticas de lean manufacturing y seis sigma como DMAIC, equipo Kaizen, control visual, SMED, 5S, etc. soportan el incremento del desempeño de las empresas.

Grimaldo-León, Silva, Fonseca y Molina [21] desarrollaron un análisis de métodos y tiempos en una empresa textil stand deportivo a través del cual pudieron diagnosticar la situación actual de este proceso e identificar posibles cuellos de botella. De igual forma, enfocándose hacia el estudio del trabajo como como herramienta para la mejora de la productividad, Mosquera, Duque y Villada [22] hicieron un estudio de métodos y tiempos en una planta de alimentos a través del cual se determinó el tiempo estándar de las actividades del proceso para la obtención de leche pasteurizada y los movimientos innecesarios realizados a través de estudio del trabajo.

Dada toda la importancia de las herramientas lean para el mejoramiento de los procesos productivos, planteada anteriormente, y su enfoque hacia la mejora de competitividad, en el presente trabajo se aplicó un estudio de métodos y tiempos, la metodología de 5S, una metodología propuesta para la evaluación de rendimientos de la materia prima y una guía para la implementación de PML en una empresa del sector maderero colombiano. Esto le 
permitió a esta incrementar la productividad para estandarizar los procesos, la determinación de un tiempo estándar realista, un mejor uso de la materia prima e insumos, así como el mitigamiento del impacto al medio ambiente.

El caso de estudio está estructurado acorde a como se describe: en el segundo apartado se muestran las generalidades del proceso productivo de fabricación de estibas, luego en el tercer apartado se describen las generalidades del estudio de métodos y tiempos, $5 \mathrm{~S}$ y una metodología para el cálculo de rendimiento de la materia prima. Finalmente, en el cuarto y quinto apartado se analizan los resultados obtenidos y se concluye mostrando los aportes a la empresa analizada.

\section{Generalidades del proceso productivo}

El proceso de elaboración de estibas de madera tiene varias etapas. Este inicia cuando el operario realiza el alistamiento de la máquina y presenta las medidas requeridas para la fabricación de la estiba solicitada. Posteriormente, se calcula el número de pies brutos requeridos multiplicado por la cantidad de estibas que se desea fabricar. En esta etapa, la madera pasa a la máquina sin fin 1 , donde es bloqueada y se le da la medida inicial de ancho para que cumpla con las especificaciones solicitadas por el cliente.

Luego, la materia prima ya dimensionada pasa a la etapa de deshilado y más concretamente a la máquina sin fin 3 , donde se verifica el dimensionado exactorequeridoparaobtenerun productoterminado conforme a las especificaciones. En esta etapa se le da espesor requerido a la tabla, lo que permite un mejor acabado a la madera ya dimensionada.

En el proceso siguiente, la madera se corta y dimensiona con ancho y espesor requeridos finales en los péndulos. Las tablas ya cortadas según la especificación pasan a la etapa de ensamble, en la cual se procede a realizar un armado de la estiba bajo las especificaciones acordadas. De ser necesario un tratamiento fitosanitario, las estibas se llevan a un horno de secado, en el cual serán almacenadas a una temperatura a $89^{\circ} \mathrm{C}$ durante 30 minutos con la finalidad de disminuir el porcentaje relativo de humedad presente en la madera hasta un valor máximo de $11 \%$ y de esta forma evitar el cre-

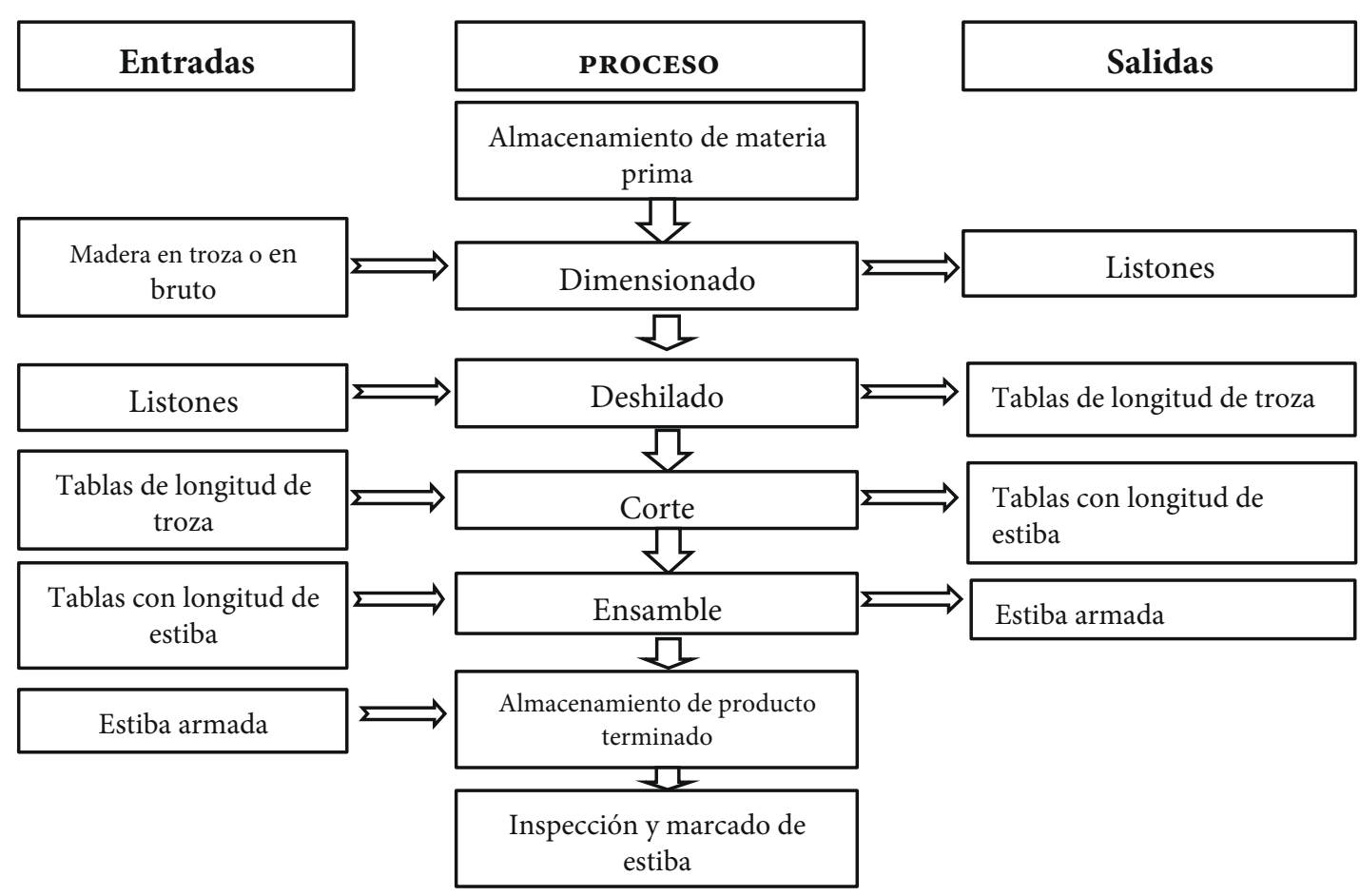

Figura 2. Descripción gráfica del proceso

Fuente: elaboración propia 
cimiento de hongos. Finalmente se aplica a las estibas un insecticida de suelo, cuyo principio activo son los clorpirifós. Por último, son transportadas a la zona de despacho o almacenamiento, donde son marcadas e inspeccionadas (figura 2).

Como unidad de estudio, se consideró la estiba sC, ya que esta cuenta con una alta participación en la demanda de la compañía (90\%). Esta estiba se encuentra estructurada por los componentes que se describen en la tabla 1 .

Tabla 1. Estructura de estiba sc $1,00 \times 1,30 \mathrm{~m}$

\begin{tabular}{|c|c|c|c|}
\hline Descripción & Medida & $\begin{array}{c}\text { N. }^{\circ} \\
\text { unidades }\end{array}$ & Convención \\
\hline Tabla superior & $2 \times 9 \times 1,30$ & 8 & TS \\
\hline Tabla inferior & $2 \times 9 \times 1,30$ & 2 & TI1 \\
\cline { 2 - 4 } & $2 \times 8 \times 82$ & 3 & TI2 \\
\hline Tabla central & $2 \times 8 \times 100$ & 3 & TC \\
\hline Taco & $8 \times 8 \times 13,5$ & 9 & TO \\
\hline
\end{tabular}

Fuente: elaborado a partir de información suministrada por la empresa

\section{Materiales y métodos}

Se detalla a continuación la metodología que se empleó en el desarrollo del presente trabajo investigativo. Se describen herramientas para mejorar la productividad, propias de la ingeniería industrial, dentro de las cuales se encuentra el estudio de métodos y tiempos, que es fundamental para la estandarización de actividades. Otra herramienta complementaria es la metodología de las 5S, que aporta al orden y limpieza de áreas, en pro de permitir que las actividades no tengan retrasos por no ubicar elementos vitales durante inicios de corrida, por ejemplo. Igual de importantes a la metodología de $5 \mathrm{~S}$ son las buenas prácticas de operación que, de la mano con parámetros de producción más limpia y una metodología para el cálculo de rendimientos y desperdicios de la madera como materia prima, pueden hacer más productiva la organización objeto de estudio.

\subsection{Estudio de métodos e implementación de $5 \mathrm{~S}$}

A través de la aplicación del estudio de métodos, se logró describir y diagramar el método de trabajo actual y propuesto. Esto se hace a partir de Cursogramas específicos como el sinóptico y analítico de material y de operario, proceso que aportó un gran valor a la empresa, ya que esta carecía de la documentación antes mencionada y sobre todo su implementación.

Una vez los métodos de trabajo fueron mejorados, se procedió a evaluar la mejora en el tiempo estándar de fabricación del producto; se empleó como herramienta vital la medición del trabajo, que es considerada como la aplicación de técnicas para determinar el tiempo que invierte un trabajador calificado en llevar a cabo una tarea definida efectuándola según norma de ejecución preestablecida [23]. Como metodología para el cálculo del tiempo estándar de la fabricación de estibas de madera se empleó la descrita por la Organización Internacional del Trabajo (ОIT), definida a partir del cálculo del tiempo observado, el tiempo normal (TN), los suplementos laborales y el factor de ritmo con el que se lleva a cabo el proceso de fabricación de estibas de madera.

Para mejorar la productividad, finalmente se propuso una metodología para calcular el rendimiento de la madera, información que es de vital importancia para determinar la productividad de las estaciones de trabajo y del sistema en general, así como de sus desperdicios. Al mismo tiempo y con un objetivo similar, se propone una guía metodológica para la implementación de un programa de producción más limpia, orientada a mejorar el uso de la materia prima a través de mejores prácticas de manufactura y concientización del personal de planta de la organización y, de este modo, reducir el porcentaje de desperdicio en las instalaciones de la empresa.

\subsection{Implementación 5S}

Takashi Osada desarrolló el concepto original de $5 \mathrm{~S}$ a principios de 1980; él introdujo el concepto como los cinco principios para un medio ambiente de calidad total [24]. Estos conceptos son totalmente aplicables a cualquier lugar de trabajo. Cada $\mathrm{S}$ tiene un significado relacionado con el sentido de utilización, clasificación, sentido de orden, sentido de aseo, sentido de logro y sentido de autodisciplina. Permite lograr buenos resultados relacionados con el orden y la limpieza a corto plazo. Esta herramienta es fundamental cuando se desea mejorar un proceso productivo, pues facilita la disminución de tiempos perdidos por desorganización. Apoya en la mejora de la productividad y el clima laboral, ya que contribuye a desarrollar buenos hábitos dentro y fuera del trabajo. Se implanta 
un programa de $5 \mathrm{~S}$ en las áreas de almacenamiento de materia prima, dimensionado, deshilado, corte de tablas, ensamble, tratamiento fitosanitario, con resultados interesantes en la cultura del orden y limpieza de las áreas. Inicialmente se procedió a realizar un diagnóstico de la situación actual con respecto al cumplimiento de ciertos aspectos de la metodología a través de un check list. A partir de ahí, se aplicaron las fases de la metodología [25]: clasificar (seiri) significa remover de nuestra área de trabajo todo lo que no se necesita para realizar las operaciones productivas; ordenar (seiton) es organizar los artículos, equipos o documentos que se necesitan para facilitar su uso e identificarlos, en forma adecuada, para localizarlos y, posteriormente, regresarlos a su lugar. Es necesario asignar un lugar específico para cada cosa u objeto, de manera que se facilite su identificación, localización y disposición. Limpiar (seiso) quiere decir mantener en buenas condiciones el equipo de trabajo y conservar limpio el medio ambiente. Estandarizar (seiketzu) es definir una manera consistente de llevar a cabo las actividades de selección, organización y limpieza, y disciplina (Shitsuke) es crear las condiciones que fomenten el compromiso de los integrantes de la organización para formar un hábito con las actividades relacionadas con las 5 S. En esta etapa se involucró, a través de capacitaciones, charlas y brigadas, a operarios, supervisores, mandos medios y gerencia general. Se sensibilizó a través de carteles y señalizaciones del programa.

\subsection{Estudio de tiempos}

Se siguió la metodología propuesta por Caso [26], denominada "De vuelta a cero" (snapback), en la cual se pulsa el botón de lectura, se registra el tiempo para el elemento $y$, automáticamente, se regresa a cero y se acumula el tiempo del siguiente elemento. Se consideró, para el cálculo del tiempo estándar, el tiempo observado, el normal, los tiempos suplementarios y el factor de ritmo de cada actividad. Se verificó la estandarización del proceso productivo a partir de la propuesta de mejora ofrecida por el estudio de métodos realizado con la finalidad de garantizar la repetitividad del estudio, y también se consideró la elección de trabajadores calificados. Posteriormente fueron realizadas veinte observaciones como premuestras, para obtener una visión del comportamiento y la variabilidad de los datos. Para exactitud en la medición, el proceso se dividió en elementos (en una o varias actividades, tabla 2).

Tabla 2. División de actividades en elementos y convención

\begin{tabular}{|c|c|c|}
\hline Elementos & Subelementos & Convención \\
\hline $\begin{array}{l}\text { Transporte de madera en } \\
\text { troza a máquina sin fin }\end{array}$ & Todos & - \\
\hline \multirow{2}{*}{$\begin{array}{l}\text { Inspección y dimensio- } \\
\text { namiento (ancho) }\end{array}$} & $\begin{array}{l}\text { Tabla superior } \\
\text { e inferior } 1\end{array}$ & TS y TI1 \\
\hline & $\begin{array}{l}\text { Tabla inferior } \\
2, \text { tabla central } \\
\text { y tacos }\end{array}$ & TI2, TC, то \\
\hline $\begin{array}{l}\text { Transportada a máquina } \\
\sin \text { fin } 2\end{array}$ & Todos & $\begin{array}{l}\text { TS,TI1, TI2, } \\
\text { TC }\end{array}$ \\
\hline $\begin{array}{l}\text { Deshilado e inspección } \\
\text { (espesor) }\end{array}$ & $\begin{array}{l}\text { Tabla central, } \\
\text { inferior } 1 \text {, } \\
\text { inferior } 2 \mathrm{y} \\
\text { central }\end{array}$ & $\begin{array}{l}\text { TS,TI1, TI2, } \\
\text { TC }\end{array}$ \\
\hline \multirow{4}{*}{$\begin{array}{l}\text { Cortado de tablas } \\
\text { (longitud) }\end{array}$} & $\begin{array}{l}\text { Tabla superior } \\
\text { e inferior } 1\end{array}$ & TS y TI1 \\
\hline & Tabla inferior 2 & TI2 \\
\hline & Tabla central & TC \\
\hline & Tacos & то \\
\hline $\begin{array}{l}\text { Transportada a área de } \\
\text { ensamble }\end{array}$ & Todos & - \\
\hline $\begin{array}{l}\text { Ensamble o armado de } \\
\text { Estibas }\end{array}$ & Todos & - \\
\hline $\begin{array}{l}\text { Transporte a zona de } \\
\text { almacenamiento de } \\
\text { producto terminado }\end{array}$ & Todos & - \\
\hline $\begin{array}{l}\text { Inspección y marcado } \\
\text { de producto terminado }\end{array}$ & Todos & - \\
\hline
\end{tabular}

Fuente: elaboración propia

\subsection{Cálculo del tamaño de la muestra}

Para llevar a cabo el estudio, se realizó un muestreo probabilístico con la técnica de muestreo aleatorio simple, utilizada para seleccionar las observaciones de cada elemento de la muestra. De igual modo, se empleó el muestreo estratificado, que consistió en fragmentar en estratos el proceso de elaboración de estibas. La unidad experimental es el tiempo del proceso de elaboración de estibas, el cual se estimó en la ecuación 1.

Número de muestras $\mathrm{n}=\frac{t^{2} \mathrm{~s}^{2}}{(e)^{2}}$ 
En la ecuación $1, n$ es el tamaño de la muestra, $s$ la desviación estándar, e el error estimado en cada elemento y $t$ es el valor de la probabilidad obtenida para una distribución $t$ de Student con $n$-1 grados de libertad y $k$ relacionado al nivel de significancia del estudio. El cálculo de tamaño de la muestra final se realizó a partir de la determinación de tamaño de la muestra para cada elemento. De cada uno de estos valores obtenidos se tomó el mayor valor, con la finalidad de ser lo más exigente posible en la exactitud del estudio.

\subsection{Valoración del factor de ritmo y los suplementos}

Se empleó una escala numérica que permitió la obtención del factor de ritmo de cada empleado con la finalidad de corregir las diferencias evidenciables en la rapidez de diferentes operarios al realizar un mismo trabajo manual, propios de la destreza desarrollada por ellos. En este caso, se empleará la escala de 75-100, que ayudará a no caer en equívocos al momento de definir el tiempo estándar de cada actividad. En la tabla 3 se muestra la valoración de cada ritmo de trabajo y su descripción aplicada.

Tabla 3. Descripción y valoración del ritmo de trabajo

\begin{tabular}{|c|c|}
\hline \multicolumn{2}{|r|}{ Escala 75-100 } \\
\hline Variación \% & Descripción \\
\hline 0 & Actividad nula \\
\hline 50 & $\begin{array}{l}\text { Muy lento, movimientos torpes, } \\
\text { inseguros. El operario parece medio } \\
\text { dormido y sin interés en el trabajo. }\end{array}$ \\
\hline 75 & $\begin{array}{l}\text { Constante, resuelto sin prisa, como } \\
\text { obrero no pagado a destajo, pero } \\
\text { bien dirigido y vigilado. Parece } \\
\text { lento, pero no pierde tiempo. }\end{array}$ \\
\hline 100 & $\begin{array}{l}\text { Activo, capaz, como de obrero } \\
\text { calificado medio, pagado a destajo. } \\
\text { Logra con tranquilidad el nivel de } \\
\text { precisión y calidad fijado. }\end{array}$ \\
\hline 125 & $\begin{array}{l}\text { Muy rápido. El operario actúa con } \\
\text { gran seguridad, destreza y coordina- } \\
\text { ción de movimientos, muy por enci- } \\
\text { ma del operario calificado medio. }\end{array}$ \\
\hline 150 & $\begin{array}{l}\text { Excepcionalmente rápido. Con- } \\
\text { centración y esfuerzo intenso sin } \\
\text { probabilidad de durar por largos } \\
\text { períodos. Actuación de virtuoso, } \\
\text { solo alcanzada por pocos trabajado- } \\
\text { res sobresalientes. }\end{array}$ \\
\hline
\end{tabular}

Los suplementos laborales se emplearon ante la necesidad del operario de realizar paradas en su trabajo para recuperarse de la fatiga producida durante el desarrollo de la tarea y la atención de sus necesidades personales. Estos periodos de inactividad, que son un porcentaje del tiempo normal, se valoraron de acuerdo con las características del trabajador y de la tarea.

Para el cálculo de los suplementos laborales, se tomó en consideración una guía evaluativa recomendada por la oit, de la cual se muestra un fragmento debido a su extensión (tabla 4).

Tabla 4. Algunos suplementos laborales recomendados por OIT

\begin{tabular}{|c|c|c|}
\hline \multicolumn{3}{|c|}{ CONSTANTES } \\
\hline \multicolumn{2}{|c|}{ A. Necesidades personales } & 5 \\
\hline \multicolumn{2}{|l|}{ B. Básico por fatiga } & 4 \\
\hline \multicolumn{3}{|c|}{ VARIABLES } \\
\hline \multicolumn{2}{|l|}{ A. Por trabajar de pie } & 2 \\
\hline \multicolumn{3}{|l|}{ B. Por postura anormal } \\
\hline \multicolumn{2}{|l|}{ Ligeramente incómodo } & 0 \\
\hline \multicolumn{2}{|l|}{ Incómodo } & 2 \\
\hline \multicolumn{2}{|l|}{ Muy incómoda } & 7 \\
\hline \multirow{14}{*}{$\begin{array}{l}\text { C. Levantamiento de } \\
\text { pesos y uso de fuerza }\end{array}$} & Peso en $\mathrm{Kg}$ & \\
\hline & 2,5 & 0 \\
\hline & 5 & 1 \\
\hline & 1,7 & 2 \\
\hline & 10 & 3 \\
\hline & 12,5 & 4 \\
\hline & 15 & 6 \\
\hline & 17,5 & 8 \\
\hline & 20 & 10 \\
\hline & 22,5 & 12 \\
\hline & 25 & 14 \\
\hline & 30 & 19 \\
\hline & 40 & 33 \\
\hline & 50 & 58 \\
\hline \multicolumn{3}{|l|}{ D. Intensidad de luz } \\
\hline \multicolumn{2}{|c|}{$\begin{array}{l}\text { Ligeramente por debajo de lo } \\
\text { recomendado }\end{array}$} & 0 \\
\hline \multicolumn{2}{|l|}{ Bastante por debajo } & 2 \\
\hline \multicolumn{2}{|c|}{ Absolutamente insuficiente } & 5 \\
\hline
\end{tabular}

Continúa en la pág. 53

Fuente: elaboración propia 
Viene de la pág. 52

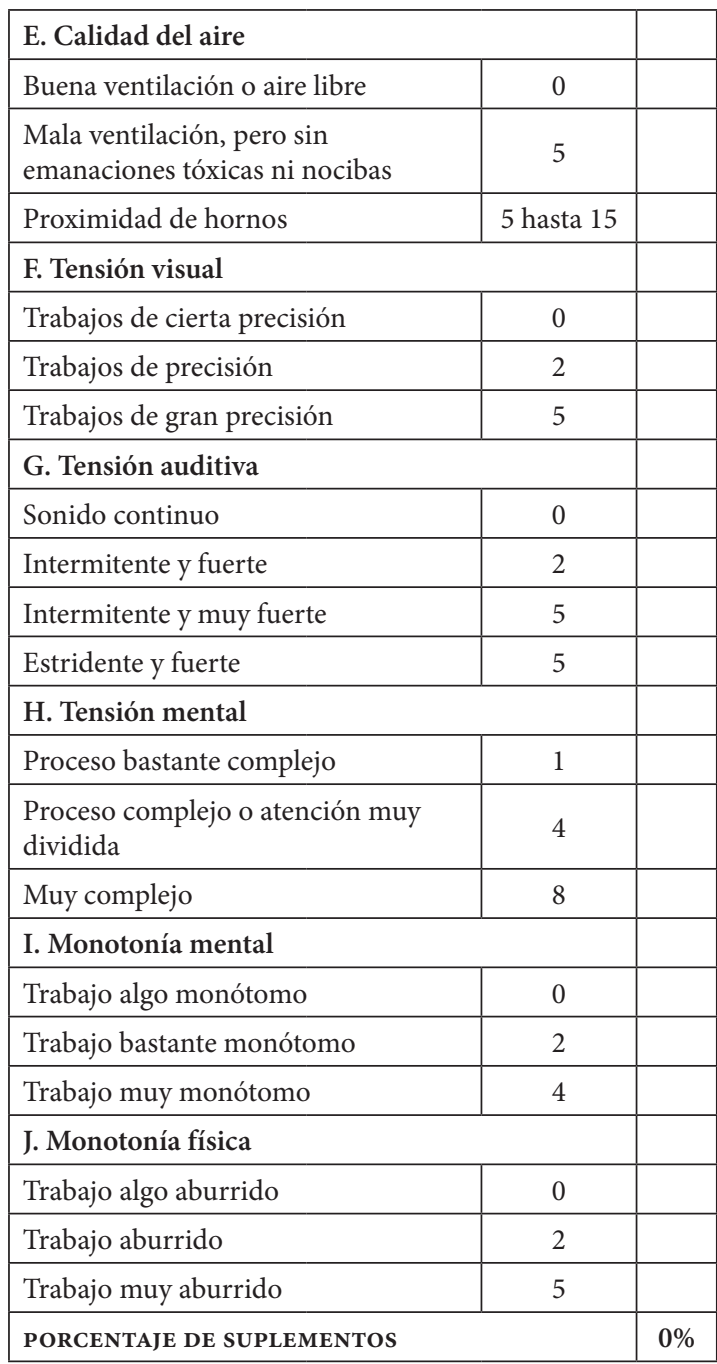

Fuente: [27]

\subsection{Obtención del tiempo estándar}

El tiempo estándar es el que un trabajador calificado y conocedor de su tarea requiere para realizarla a un ritmo normal. Para su cálculo, primeramente, se determinó el tiempo reloj, que es el tiempo que invierte el operario para realizar la tarea encomendada y que se mide mediante un cronómetro (observaciones halladas en cada elemento). En paralelo, mientras se determinaba el tiempo reloj, se calificó muy detalladamente la actividad realizada por el operario a través del factor de ritmo (FR). Este concepto surge de la necesidad de corregir las diferencias que se producen al existir trabajadores rápidos, normales y lentos al realizar una misma tarea. Con el tiempo reloj determinado con sus respectivos FR, se calculó el tiempo normal, obtenido de multiplicar el tiempo observado por su respectiva calificación o FR. Se evaluaron posteriormente los suplementos laborales, ya que es preciso que el operario realice paradas en su trabajo para recuperarse de la fatiga producida al realizar la tarea y atender a sus necesidades personales. Estos periodos de inactividad, que son un porcentaje del TN, se valoraron de acuerdo con las características del trabajador y de la tarea. Finalmente, se calculó el tiempo estándar como el resultado de la multiplicación del tiempo normal por el porcentaje añadido de suplementos laborales.

\subsection{Metodología para la medición de rendimientos}

Esta metodología consiste en medir porcentualmente el rendimiento real de la madera, así como los desperdicios generados. Para la empresa analizada estos valores son de vital importancia, ya que a partir de ellos se controla adecuadamente la producción y la eficiencia del sistema, en pro de la mejora de la productividad. Esta metodología parte de la premisa de que las trozas de madera llegan desconchadas y listas para aserrar, lo que facilita la medición del rendimiento. El cálculo se basa en la cubicación de la troza entregada a producción y posterior cubicación de la madera aserrada, para luego calcular los rendimientos y desperdicios. Esta cubicación se hace a partir del volumen de la madera que llega en listones ortoédricos. Para cubicar las trozas, se empleó la ecuación 2.

$V=0,7854 * \frac{(D+d)^{2}}{2} * L$

En la ecuación 2, $V$ es el volumen en $\mathrm{m}^{3}, D$ es el diámetro mayor en metros, $d$ es el diámetro menor en metros y $L$ es la longitud en metros.

Luego se cubica la madera aserrada con base en la fórmula 3.

$V=\frac{L^{*} A^{*} E}{12}$

En la ecuación 3, $V$ es el volumen en pies tablares, $L$ es la longitud de la tabla en pies, $A$ es el ancho de la tabla en pulgadas y $E$ es el espesor de la tabla en pulgadas. 
Para obtener el rendimiento en porcentajes, se aplicará la siguiente relación: el volumen en tablas generalmente se obtiene en pies tablares; por ello, se realizará la transformación del volumen de madera aserrada en las mismas unidades cúbicas. Para esto, se considera la equivalencia de $1 \mathrm{~m}=424 \mathrm{pt}$.

A partir de los anteriores cálculos, se determinó el rendimiento de la madera con la ecuación 4 .

$R=\frac{\text { volumen en tablas en } \mathrm{m}^{3}}{\text { volumen en trozas en } \mathrm{m}^{3}}$

\section{Resultados}

A continuación, se presentan los resultados de la investigación conformada por el estudio de métodos y tiempos, cálculos para el rendimiento de la madera, $5 S$ y guía de PML.

\subsection{Estudio de métodos}

El proceso de fabricación de las estibas de madera sigue un proceso de manufactura tipo flow shop organizado en forma de $\mathrm{U}$, en el cual las máquinas se encuentran ubicadas en una secuencia lógica acorde a las etapas del proceso. En este no se recorren distancias extensas, los procesos son dependientes, los niveles de producción son altos, siendo en este sistema productivo el principal producto la estiba de madera sc. Por esta razón, fue seleccionado el proceso de elaboración de dicho producto para su análisis y mejora.

Se caracterizaron los procesos; se realizaron diferentes cursogramas y diagramas, y se propusieron e implementaron mejoras al proceso. Se redujo una operación (-33\%), un transporte (-25\%) sobre el material; hubo una mejora del $25 \%$ en la distancia recorrida en metros, representada en $5 \mathrm{~m}$ de recorrido. Una vez definidas las mejoras, se logró finalmente la estandarización y mejora del proceso; se lograron tiempos de producción uniformes que facilitaron tener una mejor planeación de la producción como insumo básico de la productividad (tabla 5).

Se verificó el cumplimiento de las acciones de mejora propuestas, lo que hizo que se llegara a un $96,5 \%$ de incremento en este indicador, cuyo resultado confirmó el aval para el desarrollo del estudio de tiempos del método actual de trabajo. Por otra

Tabla 5. Cursograma analítico elaboración estiba de madera

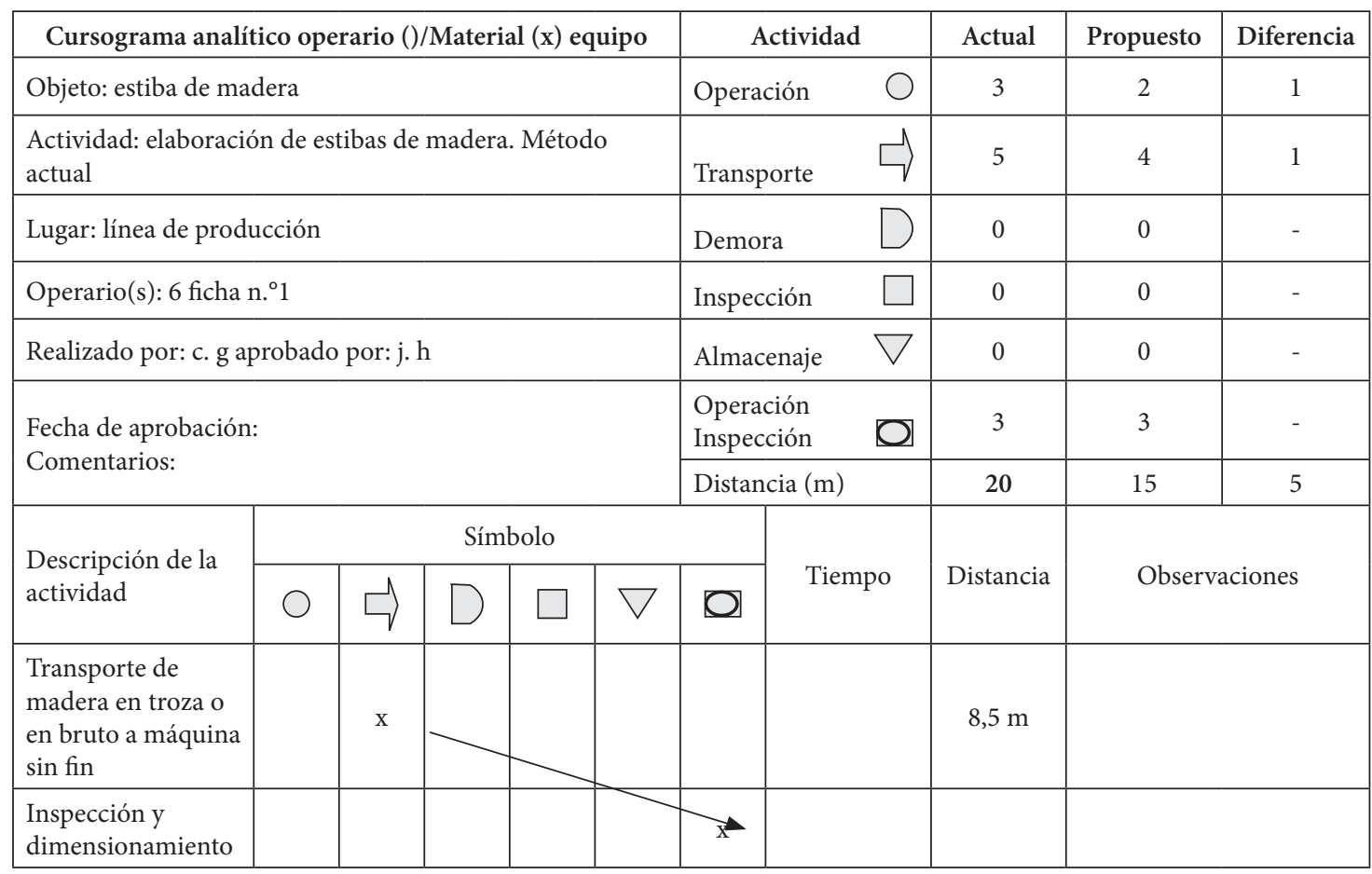

Fuente: elaboración propia 
parte, en las auditorías realizadas al proceso, se vio desorganización en las zonas de trabajo, la no clasificación de desechos y la ausencia de buenas prácticas de manufactura. Por esta razón, se aplicó una lista de chequeo que permitiera ayudar en el diagnóstico del estado actual de la empresa para aplicar la metodología de las $5 \mathrm{~S}$, que mostró que 4 de las $5 \mathrm{~S}$ tienen puntajes bajos, que oscilan entre 0 y 3 ; solo la $S$ de organizar obtuvo un puntaje de cumplimiento medio con 3,6. Por ende, no hubo calificaciones en la escala de 4 alto cumplimiento y 5 total cumplimiento (figura 3).

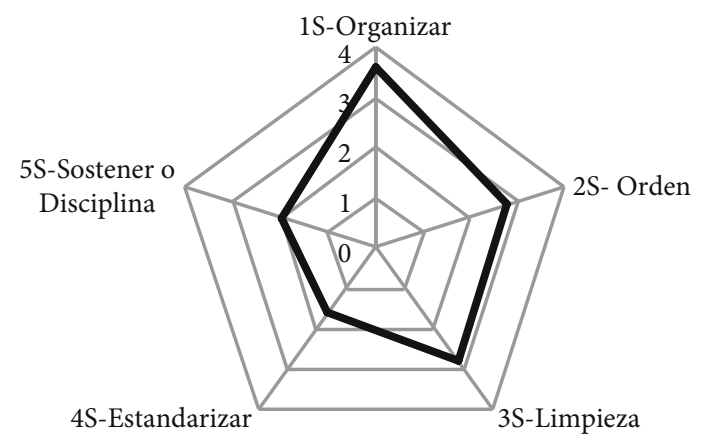

Figura 3. Resultado de diagnóstico de las $5 \mathrm{~S}$

Fuente: elaboración propia

Luego de analizar los aspectos diagnosticados, se implementaron las siguientes acciones de mejora. Se ubicaron en su sitio todas las herramientas en desuso, y este lugar fue permanente. Se rotularon los artículos según el tipo de necesidad y los innecesarios guardados o desechados. Se adoptó la política de que luego de cada actividad los operarios debían mantener todos los espacios limpios, al igual que las máquinas durante y al finalizar su actividad. Se realizaron capacitaciones para la mejora de la cultura de limpieza y orden. Fueron colocadas en diferentes lugares de las instalaciones de la planta mensajes alusivos a 5S. Se colocó en marcha la generación de ideas de mejora por parte de todos los empleados, a través de buzón de sugerencias.

Por último, luego de implantar las 5 S, se vio un cambio cultural hacia el orden y la limpieza, y un mejor ambiente laboral, sustentable en los resultados obtenidos en cada una de las áreas de procesos (figuras 4 y 5).
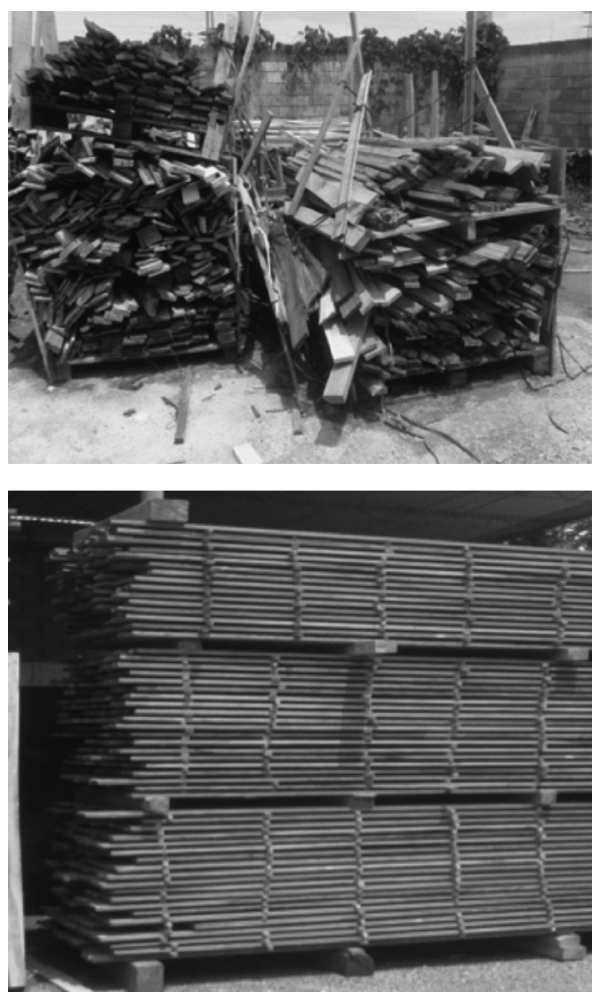

Figura 4. Producto en proceso antes y después de $5 \mathrm{~S}$ Fuente: elaboración propia
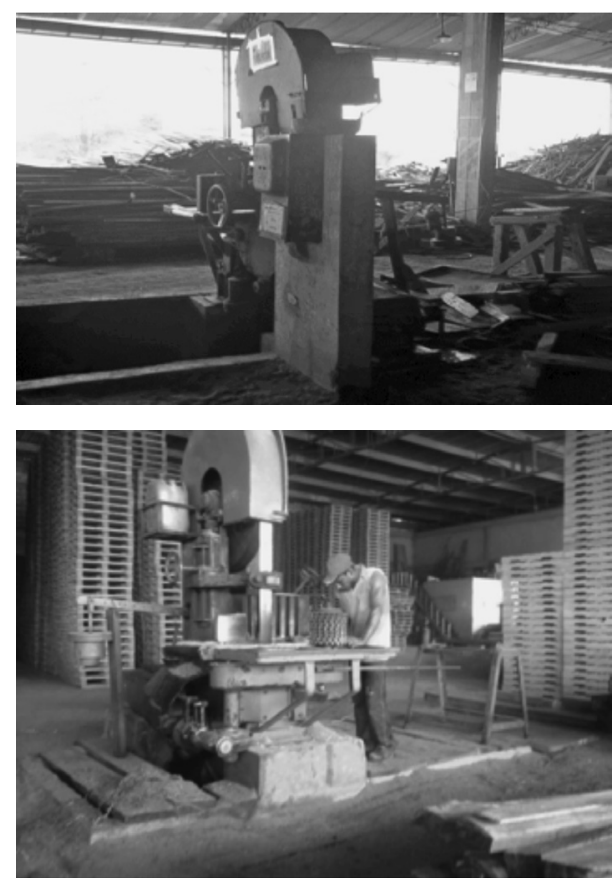

Figura 5. Proceso de deshilado antes y después de $5 \mathrm{~S}$ Fuente: elaboración propia 


\section{Estudio de tiempos}

Se realizó una toma inicial de datos que sustentaron la base de datos para el desarrollo y análisis el estudio de tiempos, y determinar el tamaño de la muestra final del mismo.

\section{Resultado de la medición previa}

Luego de hacer la toma inicial de tiempos observados durante 20 ciclos al proceso de realización de las estibas de madera, se determinó, de acuerdo con los cálculos realizados, la necesidad de tomar 33 ciclos de observaciones, razón por la cual fueron tomados 13 ciclos adicionales de observaciones (tabla 6).

Tabla 6. Determinación de tamaño de muestras o número de ciclos

\begin{tabular}{|c|c|c|c|c|c|c|c|c|}
\hline ACTIVIDADES & $\begin{array}{c}\sum \text { de los } \\
\text { tiempos } \\
\text { de los } \\
\text { elementos }\end{array}$ & tiempo^${ }^{\wedge} 2$ & $\begin{array}{c}\text { TMO } \\
\text { Tiempo } \\
\text { medio } \\
\text { observado }\end{array}$ & $\mathbf{s}$ & $\begin{array}{l}\mathrm{CV}=\mathrm{s} / \mathrm{TMO} \\
\text { Coeficiente de } \\
\text { variación }\end{array}$ & $\mathbf{t}$ & $\mathbf{k}$ & $\mathbf{n}$ \\
\hline $\begin{array}{l}\text { Transporte de madera } \\
\text { en troza o en bruto a } \\
\text { maquina sin fín }\end{array}$ & 23,70 & 28,46 & 1,19 & 0,14 & 0,12 & 2,09 & 0,06 & 29 \\
\hline \multirow{2}{*}{$\begin{array}{l}\text { Inspeccion y } \\
\text { dimensionamiento } \\
\text { (Ancho) }\end{array}$} & 3,42 & 0,59 & 0,17 & 0,01 & 0,08 & 2,09 & 0,01 & 33 \\
\hline & 10,86 & 5,99 & 0,54 & 0,07 & 0,13 & 2,09 & 0,03 & 25 \\
\hline $\begin{array}{l}\text { Transportada a } \\
\text { maquina sin fin } 2\end{array}$ & 14,70 & 10,93 & 0,74 & 0,08 & 0,11 & 2,09 & 0,03 & 31 \\
\hline $\begin{array}{l}\text { Deshilado e } \\
\text { inspección (Espesor) }\end{array}$ & 3,71 & 0,70 & 0,19 & 0,02 & 0,12 & 2,09 & 0,01 & 21 \\
\hline \multirow{4}{*}{$\begin{array}{l}\text { Cortado de tablas } \\
\text { (Longitud) }\end{array}$} & 0,95 & 0,05 & 0,05 & 0,01 & 0,13 & 2,09 & 0,003 & 28 \\
\hline & 1,05 & 0,06 & 0,05 & 0,01 & 0,12 & 2,09 & 0,003 & 18 \\
\hline & 0,84 & 0,04 & 0,04 & 0,005 & 0,11 & 2,09 & 0,002 & 25 \\
\hline & 18,07 & 16,46 & 0,90 & 0,090 & 0,10 & 2,09 & 0,035 & 27 \\
\hline $\begin{array}{l}\text { Transportada a area } \\
\text { de ensamble }\end{array}$ & 11,55 & 6,74 & 0,58 & 0,06 & 0,10 & 2,09 & 0,030 & 17 \\
\hline $\begin{array}{l}\text { Emsable o armado de } \\
\text { estibas }\end{array}$ & 54,10 & 148,09 & 2,71 & 0,30 & 0,11 & 2,09 & 0,120 & 28 \\
\hline $\begin{array}{l}\text { Transportadas a zona } \\
\text { de almacenamiento de } \\
\text { producto terminado }\end{array}$ & 43,65 & 95,62 & 2,18 & 0,14 & 0,06 & 2,09 & 0,060 & 22 \\
\hline $\begin{array}{l}\text { Inspección y } \\
\text { marcado de producto } \\
\text { terminado }\end{array}$ & 26,00 & 34,11 & 1,30 & 0,13 & 0,10 & 2,09 & 0,050 & 28 \\
\hline
\end{tabular}

Fuente: elaboración propia

\section{Factor de ritmo}

A cada uno de los tiempos observados, en los 33 ciclos, se le determinó su respectiva calificación de factor de ritmo. Los resultados resumidos se detallan en la tabla 7.
Tabla 7. Calificación de factor ritmo por elemento

\begin{tabular}{|c|l|c|}
\hline N. & \multicolumn{1}{|c|}{ Actividades } & FRM(\%) \\
\hline 1 & $\begin{array}{l}\text { Transporte de madera en troza o en } \\
\text { bruto a máquina sin fin }\end{array}$ & 95,61 \\
\hline \multirow{2}{*}{2} & Inspección y dimensionamiento (ancho) & 94,39 \\
\cline { 2 - 3 } & & 96,21 \\
\hline
\end{tabular}

Continúa en la pág. 56 
Viene de la pág. 57

\begin{tabular}{|c|l|c|}
\hline N. $^{\circ}$ & \multicolumn{1}{|c|}{ Actividades } & FRM(\%) \\
\hline 3 & Transportada a máquina sin fin 2 & 97,88 \\
\hline 4 & Deshilado e inspección (espesor) & 98,64 \\
\hline \multirow{2}{*}{5} & Cortado de tablas (longitud) & 105,61 \\
\cline { 3 - 3 } & & 86,67 \\
\cline { 3 - 3 } & & 92,12 \\
\hline 7 & Transportada a área de ensamble & 87,12 \\
\hline 8 & $\begin{array}{l}\text { Transportadas a zona de almacena- } \\
\text { miento de producto terminado }\end{array}$ & 96,52 \\
\hline 9 & $\begin{array}{l}\text { Inspección y marcado de producto } \\
\text { terminado }\end{array}$ & 94,09 \\
\hline
\end{tabular}

Fuente: elaboración propia

\section{Resultado del tiempo estándar}

Luego de aplicar la lista para evaluar las holguras relacionadas, se determinaron los suplementos laborales para cada elemento de la actividad, considerando la totalidad de los 33 considerados. Estos se obtuvieron luego de un análisis detallado de las actividades a partir de la observación directa y las métricas definidas para tal fin (tabla 8).

Tabla 8. Resumen suplementos laborales obtenidos

\begin{tabular}{|c|l|c|}
\hline \multirow{2}{*}{ N. $^{\circ}$} & \multicolumn{1}{|c|}{ ACTIVIDADES } & $\begin{array}{c}\text { \% SUPLE- } \\
\text { MENTO }\end{array}$ \\
\hline 1 & $\begin{array}{l}\text { Transporte de madera en troza o en } \\
\text { bruto a máquina sin fin }\end{array}$ & 0,16 \\
\hline 2 & $\begin{array}{l}\text { Inspección y dimensionamiento } \\
\text { ancho) }\end{array}$ & 0,44 \\
\cline { 3 - 3 } & Transportada a máquina sin fin 2 & 0,44 \\
\hline 4 & Deshilado e inspección (espesor) & 0,16 \\
\hline \multirow{2}{*}{5} & Cortado de tablas (longitud) & 0,27 \\
\cline { 3 - 3 } & & 0,28 \\
\hline 6 & Transportada a área de ensamble & 0,28 \\
\hline 7 & Ensamble o armado de estibas & 0,28 \\
\hline 8 & $\begin{array}{l}\text { Transportadas a zona de almacena- } \\
\text { miento de producto terminado }\end{array}$ & 0,28 \\
\hline 9 & $\begin{array}{l}\text { Inspección y marcado de producto } \\
\text { terminado }\end{array}$ & 0,16 \\
\hline
\end{tabular}

Fuente: elaboración propia
A partir de los datos obtenidos, como lo son el tiempo medio observado, las calificaciones y los suplementos laborales, se estimó el tiempo estándar de la operación. Se determinó que el transporte de la madera en troza a la máquina sin fin tarda 1 minuto con 18 segundos. De igual forma, se calculó que el dimensionamiento e inspección de una tabla demora aproximadamente 59 segundos. En el deshilado tarda 14 segundos y durante una hora el operario está en capacidad de deshilar 251 tablas. El péndulo encargado del corte de las tablas invierte 4 segundos en realizar el corte de aproximadamente 5 de ellas. En esta operación se obtiene un mayor número de tablas, debido a que estas se organizan en lote realizando un solo corte. En el ensamble, un operario tarda en armar una estiba 3 minutos con 22 segundos, es decir, en una hora un operario ensambla 18 estibas, para un total de 144 durante su jornada laboral (tabla 9).

Luego de realizar el cálculo de rendimiento de la madera en troza y aserrada, bajo la metodología propuesta en la muestra de 20 unidades de troza, se pudo estimar un rendimiento del 23,73\%. En comparación con la forma de realizar la estimación por parte de la empresa objeto de estudio, se mejoró la exactitud en un $15 \%$.

\section{Discusión y conclusión}

La integración global de estudio de métodos y tiempos, metodología para el cálculo de rendimiento de materia prima, la herramienta de las $5 \mathrm{~S}$, con el ciclo de mejoramiento continuo del lean manufacturing, muestra el desarrollo de un proceso interesante, que apuntó al mejoramiento de la productividad en una PYME en Colombia, empleando herramientas poco comunes en empresas de este tamaño, con resultados muy positivos en términos de productividad.

La estrategia de operaciones de la compañía debe ir ligada a su estrategia organizacional, y dentro de esta estrategia de operaciones debe resaltarse la necesidad de contar con una estrategia flexible de producción que sea capaz de generar procesos y productos innovadores, que a su vez desarrollen la productividad de las industrias.

Una adecuada selección de herramientas para la mejora, dentro de la inmensidad de posibilidades que ofrece la filosofía lean manufacturing, es un factor clave de éxito empresarial. Dentro de esta 
variedad de aplicaciones resalta por su efectividad la ingeniería de métodos y tiempos, que genera un incremento aproximado de 20 a $50 \%$ en la productividad global en empresas donde se ha aplicado.

A partir del estudio de métodos y tiempos se logró estandarizar el proceso productivo de fabricación de estibas de madera. Esto se hizo a través de la realización de diferentes cursogramas y diagramas, inexistentes en la organización hasta ese momento. Se disminuyó el 33\% de las operaciones, el $25 \%$ de los transportes y $25 \%$ en la distancia recorrida por el operario, representado como es natural en una mejora en el tiempo estándar y de la capacidad instalada.

Como resultado de la aplicación de un estudio de tiempos, se determinó el tiempo estándar de la operación de fabricación de estibas de madera en 11:42 minutos. Este estándar fue calculado inicialmente de manera incorrecta por parte de la organización, al omitir la variable suplementos laborales y la tasa promedio de desempeño, con lo cual se afectó el clima laboral, al establecer un estándar más exigente en un $30 \%$ aproximadamente.

Se dejó en evidencia la importancia de la definición adecuada de los suplementos en la productividad y definición de indicadores de eficiencia de la planta y su relación con el clima laboral.
Al aplicar una de las herramientas lean al proceso de fabricación de estibas de madera, como lo es la metodología de las $5 \mathrm{~S}$, esta aportó a la mejora de las eficiencias de los procesos productivos, ya que no se volvieron a generar retrasos por pérdida o no ubicación de herramientas y equipos necesarios tanto al iniciar la jornada como en cambios de pedido.

El evaluar una nueva forma para la determinación del rendimiento de la madera y en troza demostró el aporte valioso al proceso en la medición del mismo, ya que la metodología empleada por la empresa descartaba variables críticas en la estimación con lo son los diámetros mayor y menor de la troza, con lo cual se planteó una mejor, más exacta en un $15 \%$. Esto repercutirá directamente en las cifras de costos y utilidades de la empresa.

Se logró mejorar la cultura de las buenas prácticas de producción, en gran parte por la socialización y entrenamientos realizados en planta que se apoyaron en la guía de producción más limpia. Los operarios mostraron mejores prácticas relacionadas con el manejo, procesamiento y disposición final de la materia prima.

Se muestra a través de la revisión literaria, la integración de lean manufacturing o sus principios con otras herramientas de corte ingenieril tales como SMED, Poka Yoke, Just in time, pero poca o casi nula

Tabla 9. Resumen cálculos tiempo estándar operación fabricación estiba de madera

\begin{tabular}{|c|c|c|c|c|c|c|c|c|c|c|c|c|}
\hline \multicolumn{13}{|c|}{ CÁLCULO TIEMPO NORMAL } \\
\hline $\mathrm{N}$ & Actividades & Elementos & Tmo & $\begin{array}{c}\text { Frm } \\
(\%)\end{array}$ & Fr & $\mathrm{Tn}$ & Suplemento & $S^{*} \mathrm{Tn}$ & $\begin{array}{l}\text { Tiempo } \\
\text { estándar }\end{array}$ & $\begin{array}{c}\text { Tiempo } \\
\text { por } \\
\text { elemento }\end{array}$ & $\begin{array}{c}\text { Tiempo } \\
\text { activi- } \\
\text { dad }\end{array}$ & $\begin{array}{l}\text { Cantidad } \\
\text { a fabricar }\end{array}$ \\
\hline 1 & $\begin{array}{l}\text { Transporte } \\
\text { de madera } \\
\text { en troza o en } \\
\text { bruto a má- } \\
\text { quina sin fin }\end{array}$ & TODOS & 1,18 & 95,61 & 0,96 & 1,12 & 0,16 & 0,18 & 1,30 & 0:01:18 & 0:01:18 & 46 \\
\hline \multirow[b]{2}{*}{2} & \multirow{2}{*}{$\begin{array}{l}\text { Inspección } \\
\text { y dimensio- } \\
\text { namiento } \\
\text { (Ancho) }\end{array}$} & TS Y TI1 & 0,17 & 94,39 & 0,94 & 0,16 & 0,44 & 0,07 & 0,23 & 0:00:14 & 0:00:59 & 62 \\
\hline & & $\begin{array}{c}\text { TI2, TC Y } \\
\text { TO }\end{array}$ & 0,54 & 96,21 & 0,96 & 0,52 & 0,44 & 0,23 & 0,75 & 0:00:45 & & \\
\hline 3 & $\begin{array}{l}\text { Transportada } \\
\text { a máquina sin } \\
\text { fin } 2\end{array}$ & TODOS & 0,74 & 97,88 & 0,98 & 0,72 & 0,16 & 0,12 & 0,84 & 0:00:50 & 0:00:50 & 72 \\
\hline 4 & $\begin{array}{l}\text { Deshilado e } \\
\text { inspección } \\
\text { (espesor) }\end{array}$ & $\begin{array}{l}\text { TS,TI1, } \\
\text { TI2, TC }\end{array}$ & 0,19 & 98,64 & 0,99 & 0,19 & 0,27 & 0,05 & 0,24 & 0:00:14 & $0: 00: 14$ & 251 \\
\hline
\end{tabular}


Viene de la pág. 58

\begin{tabular}{|c|c|c|c|c|c|c|c|c|c|c|c|c|}
\hline \multicolumn{13}{|c|}{ CÁLCULO TIEMPO NORMAL } \\
\hline $\mathrm{N}$ & Actividades & Elementos & Tmo & $\begin{array}{l}\text { Frm } \\
(\%)\end{array}$ & $\mathrm{Fr}$ & Tn & Suplemento & $\mathrm{S}^{\star} \mathrm{Tn}$ & $\begin{array}{l}\text { Tiempo } \\
\text { estándar }\end{array}$ & $\begin{array}{c}\text { Tiempo } \\
\text { por } \\
\text { elemento }\end{array}$ & $\begin{array}{c}\text { Tiempo } \\
\text { activi- } \\
\text { dad }\end{array}$ & $\begin{array}{l}\text { Cantidad } \\
\text { a fabricar }\end{array}$ \\
\hline \multirow{4}{*}{5} & \multirow{4}{*}{$\begin{array}{l}\text { Cortado de } \\
\text { tablas (Lon- } \\
\text { gitud) }\end{array}$} & TS Y TI1 & 0,05 & 105,61 & 1,06 & 0,05 & 0,28 & 0,01 & 0,06 & 0:00:04 & 0:00:15 & 240 \\
\hline & & TI2 & 0,05 & 86,67 & 0,87 & 0,05 & 0,28 & 0,01 & 0,06 & 0:00:04 & & \\
\hline & & $\mathrm{TC}$ & 0,04 & 92,12 & 0,92 & 0,04 & 0,28 & 0,01 & 0,05 & 0:00:03 & & \\
\hline & & TO & 0,09 & 87,12 & 0,87 & 0,08 & 0,28 & 0,02 & 0,10 & 0:00:04 & & \\
\hline 6 & $\begin{array}{l}\text { Transportada } \\
\text { a área de } \\
\text { ensamble }\end{array}$ & TODOS & 0,58 & 96,52 & 0,97 & 0,56 & 0,16 & 0,09 & 0,65 & 0:00:39 & 0:00:39 & 93 \\
\hline 7 & $\begin{array}{l}\text { Ensamble o } \\
\text { armado de } \\
\text { estibas }\end{array}$ & TODOS & 2,71 & 99,70 & 1,00 & 2,70 & 0,27 & 0,73 & 3,43 & 0:03:22 & 0:03:22 & 17 \\
\hline 8 & $\begin{array}{l}\text { Transporte a } \\
\text { zona de alma- } \\
\text { cenamiento } \\
\text { de producto } \\
\text { terminado }\end{array}$ & TODOS & 2,18 & 94,09 & 0,94 & 2,05 & 0,16 & 0,33 & 2,38 & $0: 02: 25$ & 0:02:25 & 25 \\
\hline 9 & $\begin{array}{l}\text { Inspección } \\
\text { y marcado } \\
\text { de producto } \\
\text { terminado }\end{array}$ & TODOS & 1,30 & 95,61 & 0,96 & 1,24 & 0,18 & 0,22 & 1,47 & 0:01:28 & 0:01:28 & 41 \\
\hline
\end{tabular}

Fuente: elaboración propia

con respecto a las empleadas en este trabajo a través del estudio de tiempos y movimientos, cálculos de rendimientos de materia prima y PML en el sector maderero, lo que deja abierta la puerta para futuros trabajos empleando por ejemplo simulación de eventos discretos para el análisis y mejora de la capacidad.

\section{Referencias}

[1] Unidad de Planificación Rural Agropecuaria, Zonificación para plantaciones forestales con fines comerciales - Colombia, escala 1:100.000. Memoria Técnica. UPRA: Bogotá D.C., 2015, p. 7. Disponible en http:// www.upra.gov.co/documents/10184/13821/Zonificaci\%C3\%B3n+para+Plantaciones+Forestales/ 985d4bad-a72a-40b4-9dad-639656b295b3

[2] DANE, "Producto Interno Bruto por ramas de actividad económica”. Disponible en http://www.dane.gov. co/index.php/estadisticas-por-tema/cuentas-nacionales/cuentas-nacionales-trimestrales.

[3] DANE, "Variación porcentual Producto Interno Bruto porramasdeactividadeconómica”.Disponibleenhttp:// www.dane.gov.co/index.php/estadisticas-por-tema/ cuentas-nacionales/cuentas-nacionales-trimestrales
[4] R. Kaplan y D. Norton, The Execution Premium. Integrando la estrategia y las operaciones para lograr ventajas competitivas, Barcelona: Planeta di Agostini, 2008, p. 15.

[5] R. Navas y A. Marbelís, “Análisis Financiero: una herramienta clave para unagestión financiera eficiente", Revista Venezolana de Gerencia, vol. 14, n. ${ }^{\circ}$ 48, pp. 606-28, 2009. Disponible en http://www. scielo.org.ve/scielo.php?script=sci_arttext\&pid $=$ S1315-99842009000400009

[6] M. Porter, “QQué es la competitividad?”, Revista Apuntes de Globalización y Estrategia, vol. 1, n. ${ }^{\circ}$, pp. 2-3, 2005. Disponible en http://www.iese.edu/es/ files/5_14558.pdf

[7] M. Rochina-Barrachina, J. Mañez y J. Sanchis-Llopis, "Process Innovation and Firm Productivity Growth”, Small Business Economics, vol. 34, n. ${ }^{\circ} 2$, pp. 147-166, 2010. doi: http://dx.doi.org/10.1007/ s11187-008-9110-5

[8] B. Urgal-González y J. M. García-Vásquez, “The Strategic Influence of Structural Manufacturing Decisions", International Journal of Operations \& Production, vol. 27, n. ${ }^{\circ} 6$, pp. 605-26, 2007. doi: http:// dx.doi. org/10.1108/01443570710750286

[9] S. Pavnascar y J. Gerhenson, "Classification Scheme for Lean Manufacturing Tools”, International 
Journal of Production Research, Vol. 41, n. ${ }^{\circ}$ 13, pp. 3075-90, 2003. doi: http://dx.doi.org/10.1080/ 0020754021000049817

[10] G. Ringen, S. Aschehoug, H. Hotskog y J. Ingvaldsen, "Integrating Quality and Lean into a Holistic Production System", CIRP 47th Conference on Manufacturing System , 2014. doi: https://doi.org/10.1016/j. procir.2014.01.139

[11] A. Cruelles, Mejora de métodos y tiempos de fabricación, Barcelona: Marcombo, 2012.

[12] J. Mohd Rohani y S. Mojib Zahraee, "Production Line Analysis Via Value Stream Mapping: A Lean Manufacturing Process of Color Industy", 2nd International Materials, Industrial, and Manufacturing Engineering Conference, 2015, p. 6. doi: https://doi. org/10.1016/j.promfg.2015.07.002

[13] S. Indrawati y M. Ridwansyah, "Manufacturing Continuos Improvement Using Lean Six Sigma:An Iron Ores Industry Case Application", Industrial Engineering and Service Science, 2015. doi: https://doi. org/10.1016/j.promfg.2015.11.072

[14] M. Jiménez, L. Romero, M. Domínguez y M. D. M. Espinosa, "5S Methodology Implementation in the Laboratories of an Industrial Engineering University School", Safety and Science, vol. 78, n. ${ }^{\circ}$ 7, pp. 163-172, 2015. doi: https://doi.org/10.1016/j.ssci.2015.04.022

[15] M. Bevilacqua, F. E. Ciarapica, I. De Sanctis, G. Mazzuto, C. Paciarotti, "A Changeover Time Reduction through an Integration of Lean Practices: A Case Study from pharmaceutical Sector", Assembly Automation, vol. 35, n. ${ }^{\circ} 1$, pp. 22-34, 2015. doi: https:// doi.org/10.1108/AA-05-2014-035

[16] S. Gupta, S. Kumar-Jain, "An Application of 5 S Concept to Organize the Workplace at a Scientific Instruments Manufacturing Company", International Journal of Lean Six Sigma, vol. 6, n. ํ 1, pp.73-88, 2015. doi: https://doi.org/10.1108/IJLSS-08-2013-0047

[17] K. Ramdass, "Integrating 5S Principles with Process Improvement: A Case Study", PICMET Conference: Management of the Technology Age, Portland, Oregon, USA, 2015, p. 1908. doi: http://dx.doi. org/10.1109/PICMET.2015.7273045

[18] U. Dombrowski y T. Mielke, "Lean Leadership fundamental principles and their application". Procedia
CIRP, 2013, p. 570. doi: https://doi.org/10.1016/j. procir.2013.06.034

[19] D. Mann, “The Missing Link: Lean Leadership", Frontiers of Health Services Management, pp. 1526, 2009. Disponible en http://www.datadriven365. com/wp-content/uploads/2013/05/The-MissingLink_Lean-Leadership_DWMann.pdf

[20] I. Alhuraish, C. Robledo y A. Kobi, "The Effective of Lean Manufacturing and Six Sigma Implementation", $6^{\text {th }}$ IESM Conference, Seville, Spain, 2015, p. 1. doi: http://dx.doi.org/10.1109/IESM.2015.7380197

[21] G. Grimaldo-León, J. Silva Rodríguez, D. Fonseca-Pedraza y J. Molina-López, "Análisis de métodos y tiempos: empresas textil Stand Deportivo", Revista i3, Investigación, Innovación, Ingeniería, vol. 2, n. ${ }^{\circ}$ 1, pp. 120-39, 2015. Disponible en http://revistasdigitales.uniboyaca.edu.co/index.php/reiv3/article/ view/77/79

[22] S. Mosquera , R. Duque y D. Villada, "Estudio de métodos y tiempos en una planta de alimentos", $R e$ vista Temas Agrarios, vol. 13, n. ${ }^{\circ}$ 2, pp. 45-55, 2008. Disponible en http://revistas.unicordoba.edu.co/ index.php/temasagrarios/article/view/669/785

[23] G. Kanawaty, Introducción al estudio del trabajo, México: Limusa-Noriega, 1997.

[24] J. Ablanedo-Rosas, B. Alidaee, J. C. Moreno y J. Urbina, "Quality Improvement Supported by the $5 \mathrm{~S}$ : An Empirical Case of Study of Mexican Organization", International Journal of Production Research, vol. 48, n. ${ }^{\circ} 23$, pp. 7063-87, 2010. doi: http://dx.doi. org/10.1080/00207540903382865

[25] R. Soto Sánchez, “El proceso de las 5’s en acción: la metodología japonesa para mejorar la calidad y la productividad de cualquier tipo de empresa", Revista Gestión y Estrategia, vol. 1, n. ${ }^{\circ}$ 31, pp. 91-94, 2007. Disponible en http://zaloamati.azc.uam.mx/ bitstream/handle/11191/4806/el-proceso-de-las-5-sen-accion-la-metodologia-japonesa-para-mejorarla-calidad-y-la-productividad-de-cualquier-tipo-deempresa.pdf? sequence $=1$ \&isAllowed $=\mathrm{y}$

[26] A. Caso Neira, Técnica de medición del trabajo. Madrid: Editorial Fundación Comfametal, 2006.

[27] B. Niebel y A. Freidvals, Ingeniería Industrial: Métodos, estándares y diseño del trabajo. Mexico: McGraw-Hill, 2015. 